\title{
A conduction velocity adapted eikonal model for electrophysiology problems with re-excitability evaluation
}

\author{
Cesare Corrado ${ }^{\mathrm{a}, *}$, Nejib Zemzemi ${ }^{\mathrm{b}, \mathrm{c}}$ \\ ${ }^{a}$ Division of Imaging Sciences 8 Biomedical Engineering, \\ King's College London, London SE17EH, United Kingdom \\ ${ }^{b}$ Inria Bordeaux Sud-Ouest, Carmen team, \\ Avenue de la Vieille Tour, Talence 33000, France \\ ${ }^{c}$ IHU-LIRYC, avenue du Haut Lévèque 33600 Pessac, France
}

\begin{abstract}
Computational models of heart electrophysiology achieved a considerable interest in the medical community as they represent a novel framework for the study of the mechanisms underpinning heart pathologies. The high demand of computational resources and the long computational time required to evaluate the model solution hamper the use of detailed computational models in clinical applications. In this paper, we present a multi-front eikonal algorithm that adapts the conduction velocity $(\mathrm{CV})$ to the activation frequency of the tissue substrate. We then couple the eikonal new algorithm with the MitchellSchaeffer (MS) ionic model to determine the tissue electrical state. Compared to the standard eikonal model, this model introduces three novelties: first, it evaluates the local value of the transmembrane potential and of the ionic variable solving an ionic model; second, it computes the action potential duration (APD) and the diastolic interval (DI) from the solution of the MS model and uses them to determine if the tissue is locally re-excitable; third, it adapts the CV to the underpinning electrophysiological state through an analytical expression of the $\mathrm{CV}$ restitution and the computed local DI. We conduct series of simulations on
\end{abstract}

\footnotetext{
* Corresponding author

Email addresses: cesare.corrado@kcl.ac.uk (Cesare Corrado), nejib.zemzemi@inria.fr (Nejib Zemzemi)
}

Preprint submitted to Journal of Medical Image Analysis

December 4, 2017 
a 3D tissue slab and on a realistic heart geometry and compare the solutions with those obtained solving the monodomain equation. Our results show that the new model is significantly more accurate than the standard eikonal model. The proposed model enables the numerical simulation of the heart electrophysiology on a clinical time scale and thus constitutes a viable model candidate for computer-guided radio-frequency ablation.

Keywords: Cardiac Electrophysiology, Multi-front eikonal model, Dijkstra algorithm, Mitchell And Schaeffer model, Clinical time scale, Conduction velocity

\section{Introduction}

The propagation of an electrical stimulus in the cardiac tissue is mathematically described by the bidomain model Tung (1978); Clements et al. (2004) that is a system of a parabolic reaction-diffusion and an elliptic PDEs describing the electrical state of an intracellular and an extracellular continuum media, separated by the cell membrane. The electrical state of the cell membrane characterising the reaction term is described by a non-linear system of ODEs that either represents the biophysical fluxes of the ion species across the cell membrane (Luo \& Rudy (1991, 1994); Ten Tusscher et al. (2004)), or that tries to reproduce the shape of the action potential in a phenomenological manner (Mitchell \& Schaeffer (2003); Aliev \& Panfilov (1996)). When intracellular and extracellular conductivity tensors are considered proportional up to a given constant, it is possible to simplify the bidomain description with the so-called monodomain approximation Clements et al. (2004). The problem is thus characterised by only a parabolic non-linear reaction-diffusion equation. Even though computationally improved, the monodomain simplification still requires solving the non-linear system that arises from the numerical discretization. Since the characteristic space and time scales of the electrophysiology are small compared to the standard left ventricular dimension and cycle the length, the computational demand of the monodomain simplification is still too high in view of 
clinical applications, where the time is one of the principal constraints. Approaches based on proper orthogonal decomposition (POD) were applied to the bidomain model, furnished good results in terms of performances Corrado et al. (2016) and allowed to cope with state corrections on electrophysiology data assimilation Corrado et al. (2015). However, the requirement of building a data set of snapshots prevents their application to domains with strong heterogeneities.

The eikonal model describes the propagation of an electrical stimulus from a region $\Gamma$ of the cardiac tissue domain $\Omega_{H}$ in terms of isochronous surfaces. Thus, the eikonal model captures the propagation of the activation front, when the propagation velocity is known. Due to the causality of the propagation front, the eikonal equation can be solved in a very efficient way, either by fast marching methods (FMM) Sethian (1996) or by Dijkstra algorithm Wallman et al. (2012). The required computational time and resources enable it to be an optimal candidate in view of clinical applications. In this work, we adopt the Dijkstra algorithm for the numerical solution of the eikonal equation, since it has been shown to be very efficient Wallman et al. (2012). We suitably modify that algorithm by taking into account of the multi-front activations Wallman et al. (2013); Pernod et al. (2011); Sermesant et al. (2007) and the CV variations related to the tissue electrophysiology. We describe the cell membrane action potential with the MS model Mitchell \& Schaeffer (2003) and we use the asymptotic expression introduced in Cain et al. (2004) to locally adapt $\mathrm{CV}$ to the heart rate. The work is organised as follows: in section 2.1, we introduce the monodomain MS ionic model and its restitution properties. In section 2.2 , we present the modified eikonal model and its algorithm. In section 2.3 , we describe the proposed eikonal MS algorithm. In section 3, we apply the proposed algorithm to a tissue slab and to a ventricular geometry obtained by CT segmentation. We show through series of simulations the accuracy of the new algorithm and its performances. 


\section{Methods}

\subsection{The Monodomain Mitchell and Schaeffer model}

The MS ionic model Mitchell \& Schaeffer (2003) is a two state variable ODE system that describes the ionic fluxes across the cell membrane with a gatedinward and an ungated outward ionic current. When incorporated into a tissue $\Omega_{H}$ with boundary $\Sigma$ and for a time interval $[0, T]$, the MS monodomain model leads to the following system:

$$
\begin{array}{lr}
A_{\mathrm{m}}\left(C_{\mathrm{m}} \partial_{t} V_{\mathrm{m}}+\beta I_{\mathrm{ion}}\left(V_{\mathrm{m}}, \mathrm{h}\right)\right)-\operatorname{div}\left(\underline{\underline{D}}_{\mathrm{m}} \underline{\nabla} V_{\mathrm{m}}\right)=A_{\mathrm{m}} I_{\mathrm{app}}, & \text { in } \Omega_{H} \times(0, T), \\
\partial_{t} \mathrm{~h}+g\left(V_{\mathrm{m}}, \mathrm{h}\right)=0, & \text { in } \Omega_{H} \times(0, T), \\
\underline{\underline{D}}_{\mathrm{m}} \nabla V_{\mathrm{m}} \cdot n=0, & \text { on } \Sigma,
\end{array}
$$$$
I_{\text {ion }}\left(V_{\mathrm{m}}, \mathrm{h}\right)=-\frac{\mathrm{h}\left(V_{\mathrm{m}}-V_{\min }\right)^{2}\left(V_{\max }-V_{\mathrm{m}}\right)}{\tau_{\text {in }}\left(V_{\max }-V_{\min }\right)^{3}}+\frac{\left(V_{\mathrm{m}}-V_{\min }\right)}{\tau_{\text {out }}\left(V_{\max }-V_{\min }\right)},
$$$$
g\left(V_{\mathrm{m}}, \mathrm{h}\right)= \begin{cases}\frac{\mathrm{h}-1}{\tau_{\text {open }}}, & \text { if } V_{\mathrm{m}} \leq V_{\text {gate }}, \\ \frac{\mathrm{h}}{\tau_{\text {close }}}, & \text { if } V_{\mathrm{m}}>V_{\text {gate }},\end{cases}
$$

where $V_{\mathrm{m}}$ is the transmembrane potential, $\mathrm{h}$ is the gating variable of the inward current, $V_{\min }, V_{\max }$ are the minimum and the maximum characteristic values of the transmembrane potential, $C_{\mathrm{m}}$ is the membrane capacitance per area unit, $\beta$ is a reaction factor, typically $\beta=C_{\mathrm{m}}\left(V_{\max }-V_{\min }\right), A_{\mathrm{m}}$ is the cell surface per volume unit, $\underline{\underline{D}}_{\mathrm{m}}$ is the bulk conductivity, $I_{\mathrm{app}}$ is an external stimulus triggering the activation, $V_{\text {gate }}$ is the gate potential where inward channels switch between open and close, and $\tau_{\text {in }}, \tau_{\text {out }}, \tau_{\text {open }}$ and $\tau_{\text {close }}$ are the 4 time constants characterising the four phases of the action potential. When the tissue is activated, the transmembrane potential shows an upstroke. For a single activation, the elapsed time such as $V_{\mathrm{m}} \geq V_{\text {gate }}$ is defined as the action potential duration (APD), while for two consecutive activations the elapsed time such as $V_{\mathrm{m}}<V_{\text {gate }}$ is defined as the diastolic interval (DI). According to Mitchell \& Schaeffer (2003), it is possible to express the $A P D$ at the $(n+1)$-th beat as a function of the $D I$ at 
the $n$-th beat, with the following leading order approximation:

$$
\begin{aligned}
& A P D_{n+1}\left(D I_{n}\right)=\tau_{\text {close }} \log \left(\frac{h\left(D I_{n}\right)}{h_{\text {min }}}\right), \\
& h_{\text {min }}=4 \frac{\tau_{\text {in }}}{\tau_{\text {out }}}, \\
& h\left(D I_{n}\right)=1-\left(1-h_{\text {min }}\right) e^{-\frac{D I_{n}}{\tau_{\text {open }}}} .
\end{aligned}
$$

To obtain restitution for $C V$, we first define the diffusivity tensor as:

$$
\sigma_{\mathrm{m}}=\frac{\underline{\underline{D}}_{\mathrm{m}}}{A_{\mathrm{m}} C_{\mathrm{m}}}
$$

with a longitudinal component $\sigma_{\mathrm{m}}^{l}$, parallel to the local fibre direction $\vec{a}$ and transversal one $\sigma_{\mathrm{m}}^{t}$, and then re-arrange it as follows:

$$
\sigma_{\mathrm{m}}=\sigma_{\mathrm{m}}^{t} \underline{\underline{I}}+\left(\sigma_{\mathrm{m}}^{l}-\sigma_{\mathrm{m}}^{t}\right) \vec{a} \otimes \vec{a}=\sigma_{\mathrm{m}}^{l}(\rho \underline{\underline{I}}+(1-\rho) \vec{a} \otimes \vec{a})=\sigma_{\mathrm{m}}^{l} \underline{\underline{D}}
$$

where $\rho=\sigma_{\mathrm{m}}^{t} / \sigma_{\mathrm{m}}^{l} \leq 1$ is the ratio between the transversal and longitudinal diffusivity components and $\underline{\underline{D}}$ is a dimensionless tensor that describes the tissue anisotropy. In Cain et al. (2004), the authors obtain an expression for the $C V$ restitution on a $1 \mathrm{D}$ filament. In this work, we use the hypothesis that the ratio between transversal and longitudinal components of the $C V$ is equal to $\rho$ and does not vary with the frequency. Thus, we characterise the longitudinal component of the $C V$ with the following expression:

$$
C V_{n+1}\left(D I_{n}\right)=\frac{1}{4}\left(3 \sqrt{h\left(D I_{n}\right)-h_{\min }}-\sqrt{h\left(D I_{n}\right)}\right) \sqrt{\frac{2 \sigma_{\mathrm{m}}^{l}}{\tau_{\text {in }}}} .
$$

Remark. In multi-dimension problems (2D, 3D), the conductivity tensor $\underline{\underline{D}}$ has two eigenvalues: the former is equal to 1 and corresponds to the conductivity in the fibre direction; the second is equal to $\rho$ that corresponds to the conductivity in the transverse direction. If $\rho=1 / 2$ for instance, the $C V$ is $\sqrt{2}$ faster than it should be in the transverse direction. We take into account this issue in the next paragraph. 


\subsection{The eikonal model for the activation}

The eikonal equation describes the propagation of an electrical wavefront generated on a region $\Gamma$ and through a non-homogeneous anisotropic continuum $\Omega_{H}$. Denoting by $T_{\text {act }}(\vec{x})$ the time at which the wavefront reaches the point $\vec{x}$ and by $F(\vec{x})$ the characteristic velocity the wavefront propagates in the medium, the eikonal equation reads as follows:

$$
\begin{cases}F \sqrt{\left(\underline{\nabla} T_{\text {act }}\right)^{\top} \underline{\underline{D}} \underline{\nabla} T_{\text {act }}}=1 & \text { on } \Omega_{H} \\ T_{\text {act }}=0 & \text { on } \Gamma\end{cases}
$$

where $\underline{\underline{D}}$ is the tensor introduced in (3) that takes into account of the propagation anisotropy. To obtain the same conduction velocity values in the eikonal model and in the monodomain Mitchell and Schaeffer model, from (4) and (5) it follows:

$$
\begin{aligned}
F & =\alpha \sqrt{\frac{2 \sigma_{\mathrm{m}}^{l}}{\tau_{\mathrm{in}}}} \\
\alpha\left(D I_{n}\right) & =\frac{1}{4}\left(3 \sqrt{h\left(D I_{n}\right)-h_{\min }}-\sqrt{h\left(D I_{n}\right)}\right)
\end{aligned}
$$

Once the space distribution $D I_{n}(\vec{x})$ is known, it is possible to determine the activation times for a particular electrical state of the medium by solving the eikonal equation (5). In this paper, the eikonal equation is solved using a Dijkstra algorithm Wallman et al. (2012) which has been modified in order to take into account of the presence of re-excitable regions Wallman et al. (2013); Pernod et al. (2011); Sermesant et al. (2007) and of the local variations on the $C V$. Differently from Wallman et al. (2013); Pernod et al. (2011), in this paper, we solve numerically the ionic MS model on each node of the graph, we use the computed solution to determine whether the point is re-excitable, its value of the $D I$ and we compute the propagation velocity of the medium from the electrophysiological state of the tissue through the DI. This has many advantages: First, it allows taking into account of the spatial heterogeneity of the parameters that characterise the ionic model and thus of their high impact on the electrical wave propagation; the heterogeneity of the parameters characterising the ionic model can be considered nodal-wise. Second, it allows determining the 
$D I$ on each node of the mesh since the transmembrane potential is computed. Third, the adaptation of the $C V$ and the re-excitability criterion allow taking into account of slowing in the propagation and of functional blocks: when the electrical wave fails to propagate in the tissue especially in the refractory period.

Following Wallman et al. (2012), we denote by $n_{0}$ the set of points belonging to $\Gamma$, by $Q$ the priority queue where a set of trial points are ordered with respect to their candidate activation time and by $P_{\text {visited }}$ the container of points with a definitely determined activation time. These variables are initialized as described in Algorithm 1.

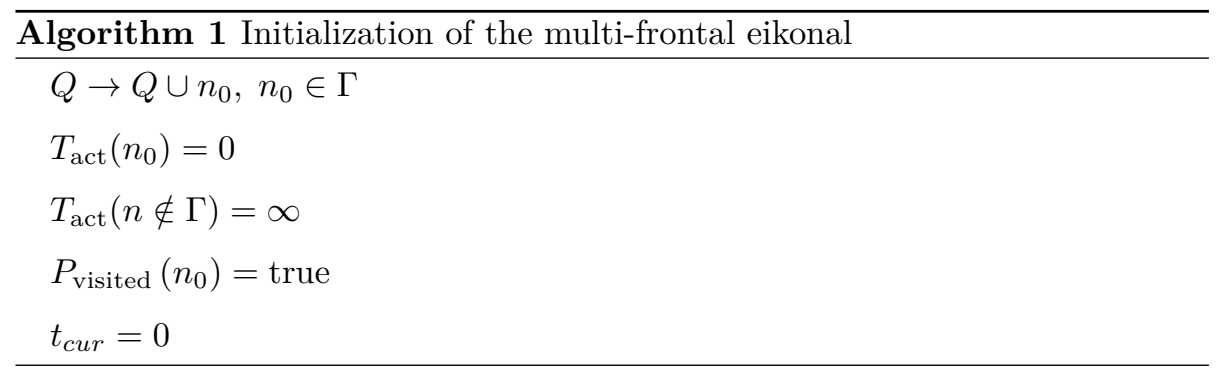

In order to compute the time that takes the electrical wave to propagate from a node $n_{i}$ characterised by its position $\vec{x}_{i}$ to a neighbour node $n_{j}$ characterised by its position $\vec{x}_{j}$, we define the cost function $c_{i j}\left(D I_{n_{i}}, D I_{n_{j}}\right)$ as follows:

$$
\left\{\begin{array}{l}
c_{i j}\left(D I_{n_{i}}, D I_{n_{j}}\right)=\sqrt{\vec{v}_{i j}^{\top} \underline{\underline{D}}^{-1} \vec{v}_{i j}} /\left(F\left(D I_{n_{i}}, D I_{n_{j}}\right) / \delta\right), \\
\vec{v}_{i j}=\vec{v}\left(n_{i}, n_{j}\right)=\vec{x}_{j}-\vec{x}_{i}
\end{array}\right.
$$

where $\delta$ is a corrective coefficient Wallman et al. (2012); Kim \& Hespanha (2003) that compensates the error introduced by the graph representation of the domain. The characteristic velocity $F\left(D I_{n_{i}}, D I_{n_{j}}\right)$ along a segment $\vec{v}_{i j}$ depends on the values of the diastolic interval at both vertices of the edge, through the parameter $\alpha\left(D I_{n_{i}}, D I_{n_{j}}\right)$. In this work, we choose the following relation:

$$
\alpha\left(D I_{n_{i}}, D I_{n_{j}}\right)=\max \left(0, \max \left(\alpha\left(D I_{n_{i}}\right), \alpha\left(D I_{n_{j}}\right)\right)\right)
$$

Introducing a time step dt, it is possible to evaluate the set of points that are activated until the time $t^{n}=n \mathrm{dt}$ and their corresponding activation time by 
applying Algorithm 2, where the function $\operatorname{TIME}\left(n_{i}, n_{j}\right)$ is defined as follows:

$$
\operatorname{TIME}\left(n_{j}, n_{i}\right)=\min \left(T_{\mathrm{act}}\left(n_{i}\right), T_{\mathrm{act}}\left(n_{j}\right)+c_{i j}\left(D I_{n_{i}}, D I_{n_{j}}\right)\right) .
$$

For each node $n$ in the graph, we denote by $\operatorname{NEIGH~}(n)$ the set of points that are connected to $n$; these points correspond to the mesh points sharing an edge with $n$.

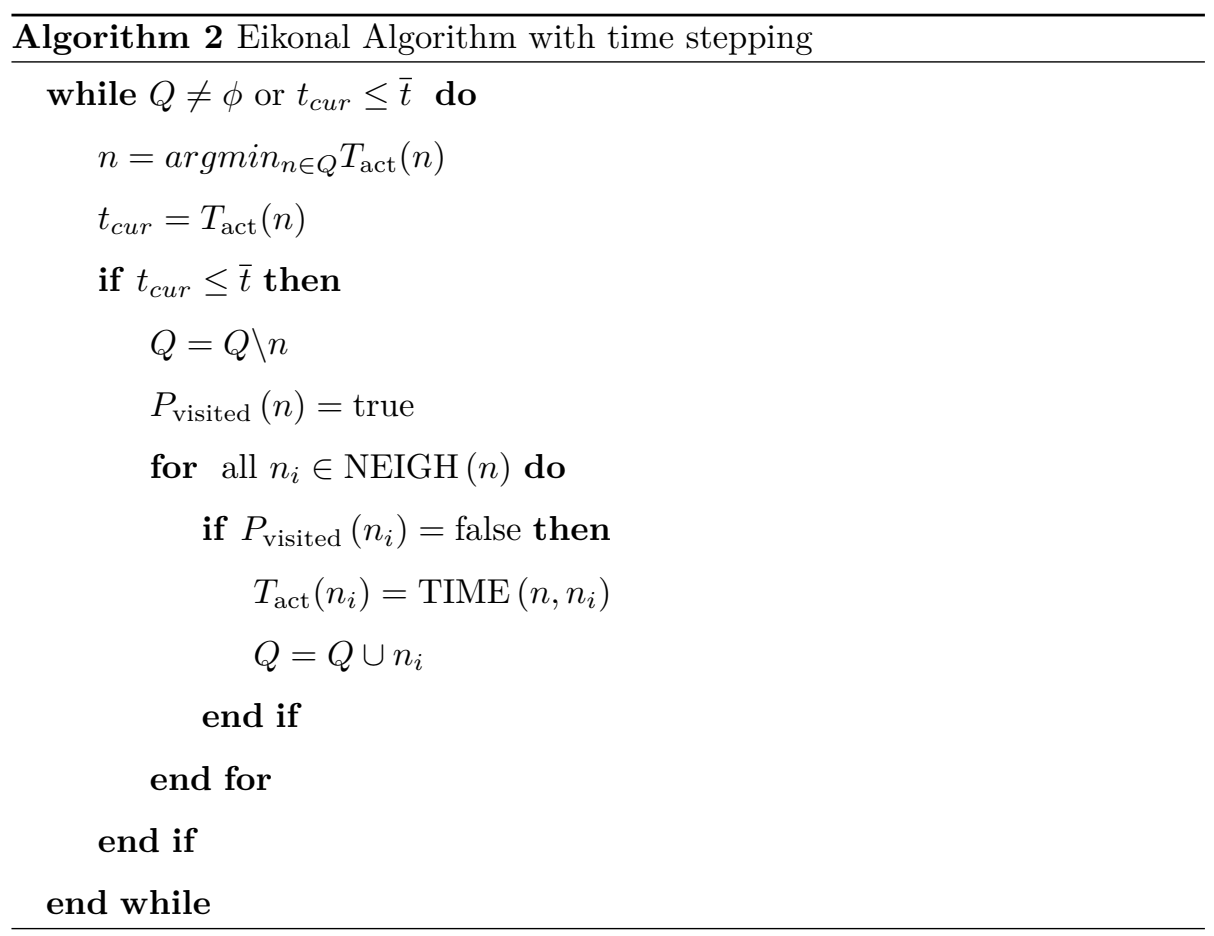

Remark. The solution of the eikonal problem with the time stepping described in Algorithm 2 allows taking into account multiple wave fronts by removing the points that became re-excitable from the container $P_{\text {visited. }}$. The same time stepping also allows to take into account the dependency of the $C V$ on the local electrophysiological state of the tissue. These variations are introduced into the model by modifying the characteristic propagation velocity $F$ that determines the cost defined in (7). 


\subsection{The eikonal Mitchell and Schaeffer model}

In this section, we present two algorithms that would be used to simulate the propagation of the electrical wave using the eikonal model: The first algorithm evaluates the state variables by solving the Mitchell and Schaeffer model at each node of the graph. This algorithm could be used in clinical applications that require the transmembrane potential, which could be the case, for instance, when electrocardiograms (ECGs) or electrograms (EGMs) need to be computed. The second algorithm adopts the leading order analytical expression of the APD restitution in order to correct the $C V$ and compute the activation times using the eikonal model, it also allows to compute pseudo-potentials as in described in Pernod et al. (2011). This algorithm could be used in clinical applications that require narrow time constraints, such as the evaluation of the outcome of a radio-frequency ablation procedure.

\subsection{Algorithm with state variables computation}

The eikonal model introduced in section 2.2 is used to mimic the conductivity of the Monodomain MS model described in section 2.1. First, we initialize the eikonal algorithm as described in Algorithm 1. We denote by $\mathrm{N}_{\mathrm{x}}$ the number of nodes in the considered graph (or mesh). For $i=1, \ldots, \mathrm{N}_{\mathrm{x}}$, the couple $\left(v_{\mathrm{m}, \mathrm{i}}^{0}, \mathrm{~h}_{\mathrm{i}}^{0}\right)$ is the initial state at the $i-t h$ node. We denote by $I_{\text {app }, \mathrm{i}}\left(T_{\mathrm{act}}^{\mathrm{i}}, t_{\mathrm{stim}}\right)$ an external electrical stimulus applied to the $i$ - th node at time $T_{\text {act }}^{\mathrm{i}}$ and with duration $t_{\text {stim }}$. The couple $\left(v_{\mathrm{m}, \mathrm{i}}^{n}, \mathrm{~h}_{\mathrm{i}}^{n}\right)$ is the electrophysiological state at time $t^{n}$ and at $i-t h$ node. The eikonal MS model is computed as described in Algorithm 3. 


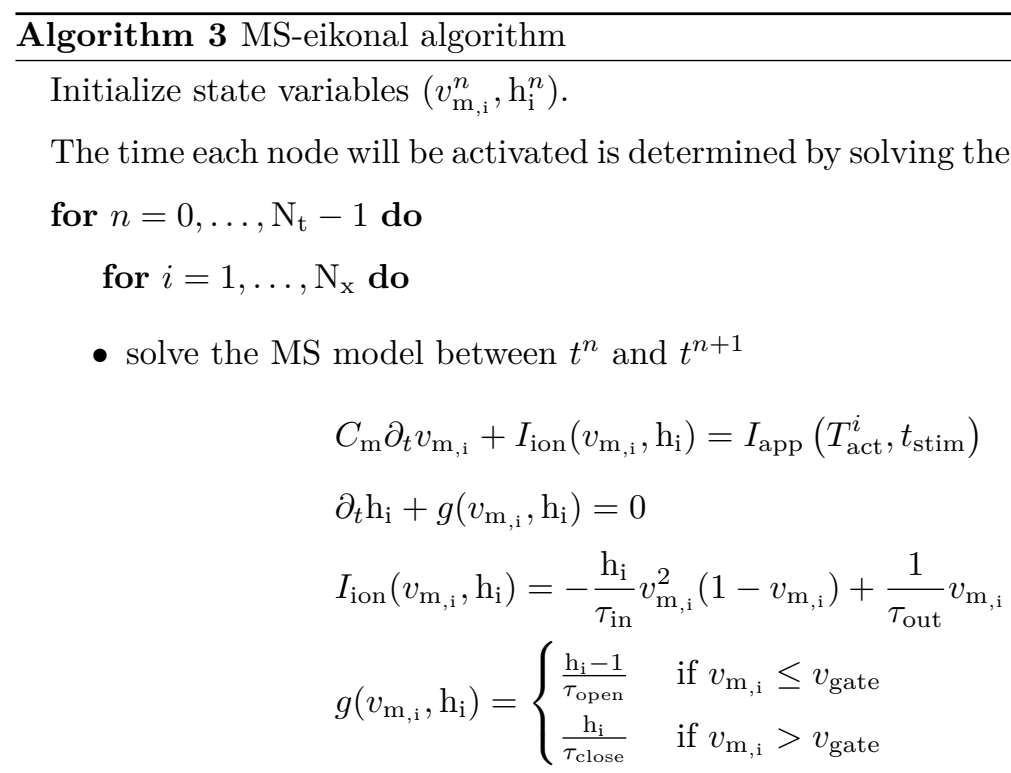

- update the local $A P D$ according to the solution $v_{\mathrm{m}_{\mathrm{i}}}$.

- If $v_{\mathrm{m}_{\mathrm{i}}} \leq v_{\text {gate }}$ and $\mathrm{h}_{\mathrm{i}} \geq h_{\mathrm{min}}$ the node is marked as re-excitable and removed from the list of known points (the values $P_{\text {visited }}\left(n_{i}\right)=$ false), the candidate activation time is set to $\infty$.

- On points that are marked as excitable, for each time the value of the $D I$ is updated as follows:

$$
D I_{\mathrm{i}}=t^{n+1}-\left(T_{\mathrm{act}}^{\mathrm{i}}+A P D_{\mathrm{i}}\right)
$$

and the value of $\mathrm{CV}$ is updated.

end for on space

Update the activation times between $t^{n}$ and $t^{n+1}$ following Algorithm 2. end for on time

On each node, the local value $A P D_{\mathrm{i}}$ of the action potential duration is updated as follows. On the $i$-th node when $t^{n+1} \geq T_{\mathrm{act}}^{\mathrm{i}}$, the flag $f_{\mathrm{dep}_{, \mathrm{i}}}$ is activated, the flag $f_{\text {rep }, \mathrm{i}}$ is deactivated, the value of $D I_{\mathrm{i}}$ is set to $0\left(D I_{\mathrm{i}}=0\right)$ and the evaluation of the repolarisation event begins for the following time iterations. In 
this paper, the repolarisation is considered occurring when the transmembrane potential crosses the threshold $v_{\mathrm{m}}=v_{\text {gate }}$ with a negative time derivative.

If repolarisation occurs at the $i$-th node, the flag $f_{\text {rep }, \mathrm{i}}$ is activated, the repolarisation time is evaluated as follows:

$$
t_{\mathrm{rep}, \mathrm{i}}=t^{n}+\frac{t^{n+1}-t^{n}}{v_{\mathrm{m}, \mathrm{i}}^{n+1}-v_{\mathrm{m}, \mathrm{i}}^{n}}\left(v_{\text {gate }}-v_{\mathrm{m}, \mathrm{i}}^{n}\right)
$$

and the local $A P D_{\mathrm{i}}$ is updated:

$$
A P D_{\mathrm{i}}=t_{\mathrm{rep}, \mathrm{i}}-T_{\mathrm{act}}^{\mathrm{i}}
$$

The flags $f_{\mathrm{dep}_{, \mathrm{i}}}$ and $f_{\mathrm{rep}_{, \mathrm{i}}}$ are then deactivated and the algorithm updates $D I_{\mathrm{i}}$ at each time iteration and until a new depolarisation occurs.

Remark. In this paper, we trigger the action potential with an applied current characterised by a constant predefined intensity $I_{\text {app }}$ and duration $t_{\text {stim. }}$. This choice allows to trigger the action potential in the full range of excitability of the ionic model Mitchell $\&$ Schaeffer (2003), provided that the intensity and the duration of the stimulus are large enough. A more complex expression of the intensity and duration of the applied stimulus can be found in the recent work of Neic et al. (2017), where the authors include a current that mimics the shape of the diffusion current.

\subsection{Algorithm with analytical restitution}

An alternative and faster formulation to Algorithm 3 is presented in Algorithm 4 . This algorithm adopts pseudo-potentials and a leading order analytical approximation for the $A P D$. The duration of the refractory period is denoted by $R P$ and evaluated as follows:

$$
R P=\tau_{\text {out }} \log \left(\frac{0.5}{v_{\text {gate }}}\right)
$$

This expression was obtained by considering that during $R P$, $\mathrm{h}<h_{\min } \ll 1$ and thus $\partial_{t} v_{\mathrm{m}} \simeq-\frac{v_{\mathrm{m}}}{\tau_{\text {out }}}$. The value of transmembrane potential at the beginning of the $R P$ here was approximated with the value $v_{\mathrm{m}}^{+}\left(h_{\min }\right)=v_{\mathrm{m}}^{-}\left(h_{\min }\right)=0.5$, 
on the null-clines, Mitchell \& Schaeffer (2003). At the end of $R P, v_{\mathrm{m}}=v_{\text {gate }}$, while $\mathrm{h}$ can be evaluated as follows:

$$
h_{\text {end,RP }}=h_{\min } \exp \left(-\frac{R P}{\tau_{\text {close }}}\right)
$$

The time lapse $T R R$ required to $\mathrm{h}$ to increase from $h_{\mathrm{end}, \mathrm{RP}}$ to $h_{\mathrm{min}}$ is evaluated from equation (1) as follows:

$$
T R R=\tau_{\text {open }} \log \left(\frac{1-h_{\text {end,RP }}}{1-h_{\text {min }}}\right)
$$

This value will be used as threshold for the diastolic interval to determine the tissue re-excitability. 


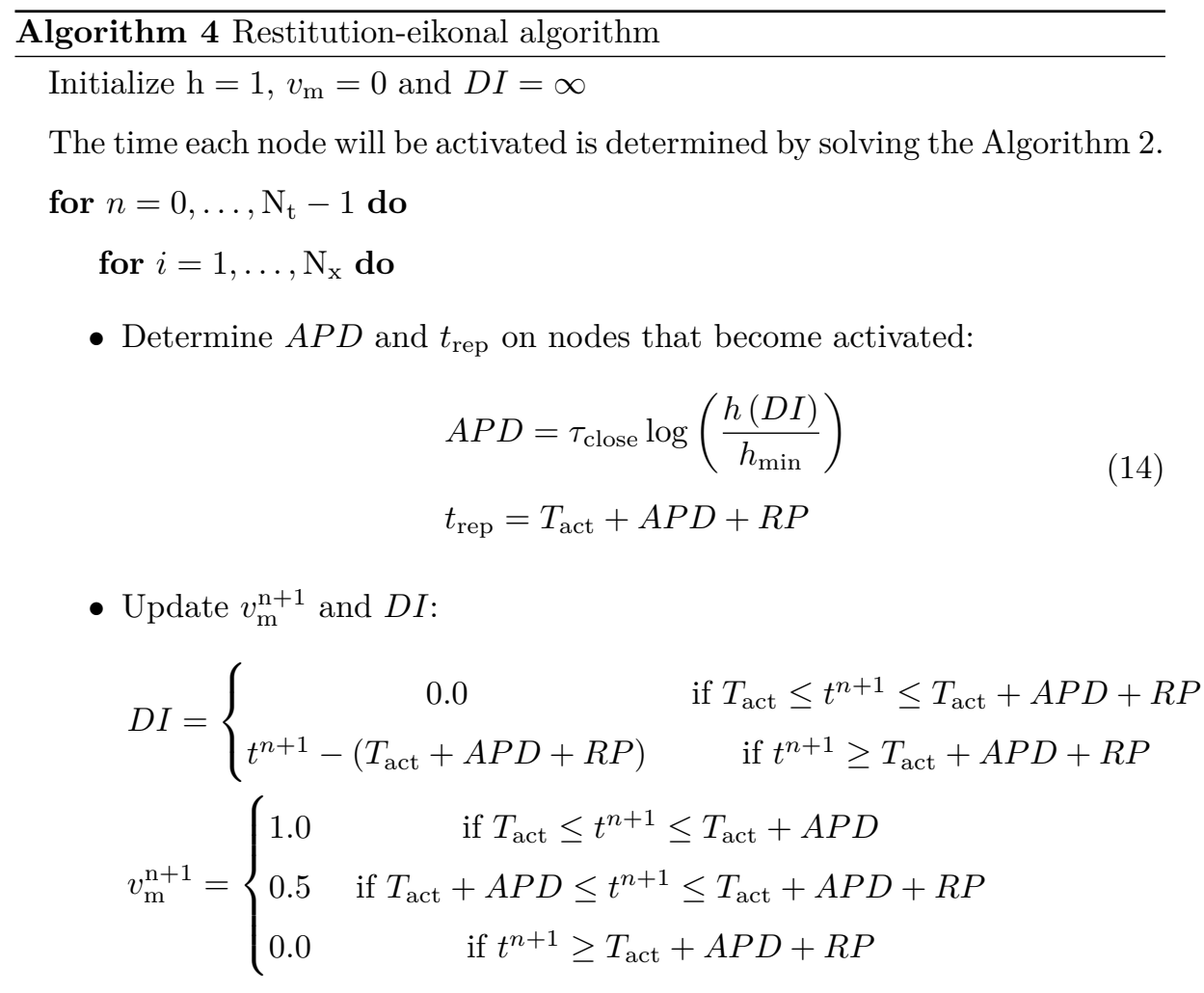

- If $D I \geq T R R$ with $T R R$ defined in (13), the node is marked as reexcitable and is removed from the list of known points (the values $P_{\text {visited }}\left(n_{i}\right)=$ false), the candidate activation time is set to $\infty$.

- On points that are marked as excitable, the value of CV is updated end for on space

Update the activation times between $t^{n}$ and $t^{n+1}$ following Algorithm 2. end for on time

\section{Numerical Results}

In this section, we compare the solutions obtained solving the eikonal MS model and the monodomain model. We use two different geometries:

- a homogeneous anisotropic tissue slab 
Table 1: Tissue properties for the MS model

\begin{tabular}{ccccc}
\hline$\tau_{\text {in }}$ & $\tau_{\text {out }}$ & $\tau_{\text {open }}$ & $\tau_{\text {close }}$ & $v_{\text {gate }}$ \\
$0.3 \mathrm{~ms}$ & $6 \mathrm{~ms}$ & $120 \mathrm{~ms}$ & $150 \mathrm{~ms}$ & 0.13 \\
\hline
\end{tabular}

- a heart geometry obtained from CT-scan segmentation

The solution of the eikonal model, obtained either with the adaptation of the $C V$ to the local electrical state or without adaptation, is compared with the solution obtained from the monodomain model. For a generic quantity $u$, the following $l_{2}$ relative error norm $\left(l_{2, \mathrm{r}}\right)$ is defined:

$$
l_{2, \mathrm{r}}\left(u^{\text {eiko }}-u^{\text {mono }}\right)=\frac{\sqrt{\frac{1}{\mathrm{~N}_{\mathrm{x}}} \sum_{i=1}^{\mathrm{N}_{\mathrm{x}}}\left(u_{i}^{\text {eiko }}-u_{i}^{\text {mono }}\right)^{2}}}{\sqrt{\frac{1}{\mathrm{~N}_{\mathrm{x}}} \sum_{i=1}^{\mathrm{N}_{\mathrm{x}}}\left(u_{i}^{\mathrm{mono}}\right)^{2}}}
$$

where $u_{i}^{\text {eiko }}$ and $u_{i}^{\text {mono }}$ are the quantities that will be compared, evaluated on the $i-t h$ computational node and obtained by solving the monodomain and the eikonal model respectively. On both examples, the monodomain problem is discretized in space with linear finite elements and in time with a first order semiimplicit scheme; the source term characterising the ionic current is treated with an ionic current interpolation, Ethier \& Bourgault (2008); Pathmanathan et al. (2012); no mass lumping is applied. On both examples, the ionic parameters characterising the MS ionic model are summarised in Table 1.

\subsection{Tissue slab}

In this section, we show the simulation results obtained on the homogeneous tissue slab depicted in Fig. 1. This simple geometry yields an easier way to compare the algorithm introduced in this paper with respect to the actual state of the art. We deal with the following 3 cases:

- A tissue slab discretised with Delaunay triangulation and fibres oriented along the $\mathrm{x}$ axis $(\vec{a}=(1,0,0))$. In this example, we show the performances of the algorithm in terms of accuracy and computational cost. 
- A tissue slab discretised with Delaunay triangulation and fibres oriented along the diagonal $\left(\vec{a}=\left(\frac{1}{\sqrt{2}}, \frac{1}{\sqrt{2}}, 0\right)\right)$ In this example, we adopt a ratio between longitudinal and transversal bulk conductivities of the order of 5 which is typical for cardiac physiology, considering the experimental conductivity values in Roberts et al. (1979). We compare the algorithm accuracy and we compare the repolarisation fronts.

- A tissue slab discretised with regular triangulation and fibres oriented along the $\mathrm{x}$ axis $(\vec{a}=(1,0,0))$. In this example, we compare algorithm 4 with algorithm 3 when the ionic parameters significantly affect the leading order approximation of the $A P D$.

\subsubsection{Horizontal fibre direction}

The homogeneous tissue slab depicted in Fig. 1 is characterised by a longitudinal conductivity $\sigma_{\mathrm{m}}^{l}=1.5 \mathrm{~cm}^{2} / \mathrm{s}$ and a transversal conductivity $\sigma_{\mathrm{m}}^{t}=$ $0.5 \mathrm{~cm}^{2} / s$; the fibres $\vec{a}$ are directed along the $x$ direction. The external stimulus is applied on the yellow region in Fig. 1 and with the intensity $I_{\text {app }}=10 \mathrm{~ms}^{-1}$. The tissue is periodically stimulated by 10 stimuli at a basic cycle length of 400 ms. The monodomain problem is discretized in space with a Delaunay triangulation with a characteristic mesh size $\mathrm{h}=215 \mu \mathrm{m}$. The number of vertices in the mesh is 325,022 and the number of tetrahedra is $1,539,099$. We use a time step $\mathrm{dt}=0.01 \mathrm{~ms}$. The eikonal model is solved on the same mesh and with the same time step, with a value $\delta=1$, so no graph related correction is introduced to the Dijkstra algorithm.

In Figure 2, two snapshots of the distribution of the transmembrane potential for both monodomain (shadowed) and eikonal model are depicted. The first snapshot is at time $20 \mathrm{~ms}$ and the second at time $52 \mathrm{~ms}$. We superimpose both solutions in order to see qualitatively how the wavefronts of both solutions behave. In Figure $3-\mathrm{A}$, we show the time course of the $l_{2, \mathrm{r}}$ error on the transmembrane potential and with respect to the monodomain solution for both the standard eikonal model (red line) and the eikonal model with the $C V$ adaptation. When the first stimulus is applied, the tissue is initially at rest, thus 


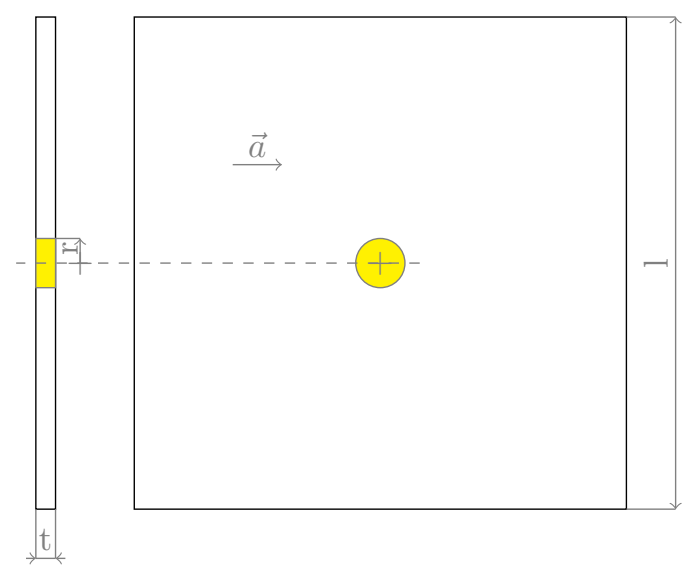

\begin{tabular}{ccc}
\hline \multicolumn{3}{c}{ Domain size } \\
\hline $\mathrm{l}$ & $\mathrm{r}$ & $\mathrm{t}$ \\
$5 \mathrm{~cm}$ & $0.25 \mathrm{~cm}$ & $0.1 \mathrm{~cm}$
\end{tabular}

Figure 1: The $5 \times 5 \times 1 \mathrm{~cm}^{3}$ tissue slab used in example 1. The yellow region corresponds to the location where the external stimulus is applied. Fibres are oriented along the $x$ direction.

$D I \rightarrow \infty$, the value of the $C V$ is equal to its maximum and both the models produce the same error. When the subsequent stimuli are applied, $\mathrm{h}<1$, the $D I$ has a finite value and the $C V$ adapts according to (6); the eikonal model with the $C V$ adaptation produces a significantly smaller error compared to the error obtained by solving the standard eikonal model. Moreover, Figure 3-A shows that the $l_{2, \mathrm{r}}$ error decreases during the propagation of the wavefront, reaching its minimum when the slab is fully depolarised. We suppose that the error increasing during the repolarization is due to the absence of the conductivity term since the eikonal model does not play any role after the depolarization; only the ionic model is solved locally at each point of the graph. Whereas, for the monodomain model we still have the influence of the electronic current represented by the diffusion term. The difference between the standard eikonal model and the eikonal model with $C V$ adaptation is much clearer when looking at the error on the activation times. Figure 3 -B shows the $l_{2, \mathrm{r}}$ error on the activation times for each of the 10 cycles. Along the 10 cycles, the mean of the $l_{2, \mathrm{r}}$ error on the activation times is $3 \%$ when using the eikonal model with $C V$ adaptation, while it is $15.5 \%$ when using the standard eikonal model: the velocity the wavefront propagates is indeed much more accurate when using a $C V$ adaptation on the eikonal model. 


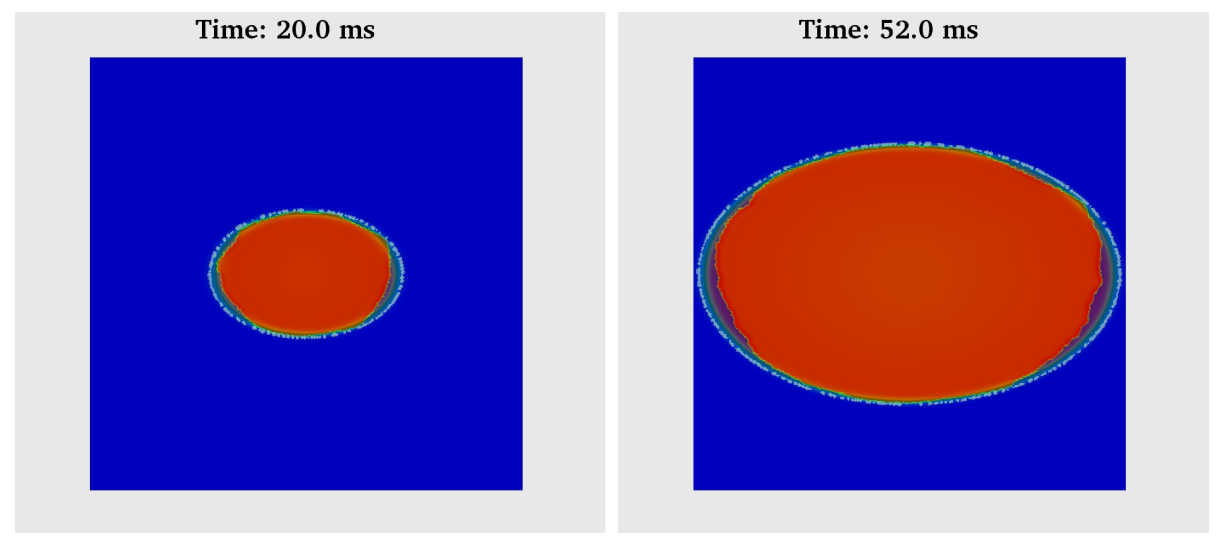

Figure 2: Plot of the eikonal and of the monodomain (shadowed) solutions for $t=20 \mathrm{~ms}$ and $t=52 \mathrm{~ms}$. The grey contour coincides with the activation front of the monodomain solution. Blue: non activated cells. Red: activated cells.

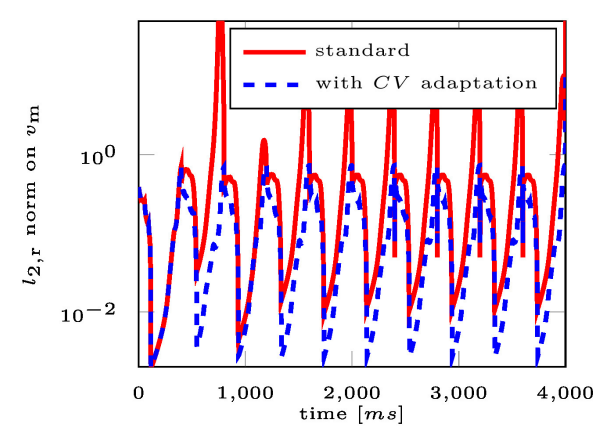

A

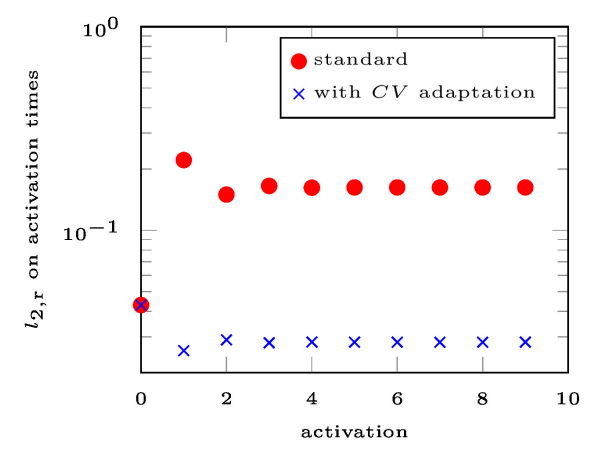

$\mathrm{B}$

Figure 3: A: Semi-logarithmic plot of the time course of the $l_{2, \mathrm{r}}$ error on the transmembrane potential for the standard eikonal model (red line) and for the eikonal model with $C V$ adaptation (blue line). B: Semi-logarithmic plot of the relative $l_{2, \mathrm{r}}$ error on the activation times at each stimulation for the standard eikonal model (red line) and for the eikonal model with $C V$ adaptation (blue dashed line). 
In Figure 4, the depolarization fronts are plotted for the monodomain model (green dots), the standard eikonal model (red dots) and the eikonal model with $C V$ adaptation (blue dots). The depolarization fronts are plotted at times $\mathrm{t}=50$, 450, 2050 and $3650 \mathrm{~ms}$. At the first cycle $(\mathrm{t}=50 \mathrm{~ms})$, the depolarization fronts of the eikonal models coincide since the tissue is initially at rest and $D I \rightarrow \infty$. From the second applied stimulus $(\mathrm{t}=450 \mathrm{~ms})$, the depolarization front of the standard eikonal model propagates faster than the monodomain depolarization front; conversely, the depolarization front of the eikonal model with $C V$ adaptation propagates at the same velocity of the monodomain depolarization front. A video showing the comparison of the depolarization fronts is available in the online supplement.

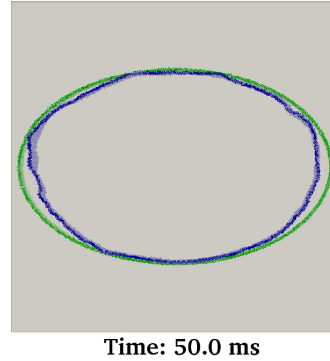

Time: $50.0 \mathrm{~ms}$

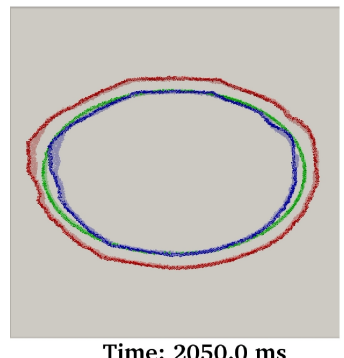

Time: $2050.0 \mathrm{~ms}$

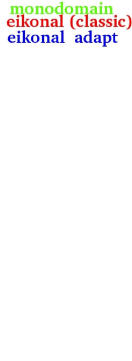
monodomain
eikonal (classic)
eikonal adapt ent

Figure 4: Comparison of wave fronts: Plot of the depolarization front of the monodomain solution (green dots), the standard eikonal solution (red dots) and the eikonal solution with CV adaptation (blue dots). The depolarization fronts are plotted at times $\mathrm{t}=50,450,2050$ and $3650 \mathrm{~ms}$. 


\subsubsection{Oblique fibre direction}

In this paragraph, we characterise the homogeneous tissue slab depicted in Fig. 1 with the fibre direction $\vec{a}=\left(\frac{1}{\sqrt{2}}, \frac{1}{\sqrt{2}}, 0\right)$, a longitudinal conductivity $\sigma_{\mathrm{m}}^{l}=5 \mathrm{~cm}^{2} / \mathrm{s}$ and a transversal conductivity $\sigma_{\mathrm{m}}^{t}=1 \mathrm{~cm}^{2} / \mathrm{s}$. This choice leads to a typical cardiac ratio between longitudinal and transversal conductivities as measured in Roberts et al. (1979). The other parameters and the space and time discretization are the same adopted in the example described in section 3.1.

The activation times following the application of the first stimulus do not differ between the eikonal model with and without CV adaptation. However, when the second stimulus is applied at $t=400 \mathrm{~ms}$ the depolarisation front of the classical eikonal model propagates faster than the depolarisation front of the monodomain, while $C V$-adapted algorithm presents the same $C V$. This difference in the propagation is present also in the subsequent stimulations of the tissue.

We also remark that the eikonal solution wave front is less accurate in the regions where the curvature is high. This behaviour is also seen in figure 4 where the fibre orientation is given by the $\mathrm{X}$-direction. This is mainly due to the accuracy of the Dijkstra algorithm in the regions where the curvature of the wave front is high and the fact that the Dijkstra solution follows the graph edges.

\subsubsection{Error on the repolarisation time}

In this section, we compare the error on the repolarisation times computed using the eikonal model with and without considering the $C V$ adaptation. We use the test case introduced in section 3.1.2 and we characterise the repolarisation time as the instant when the transmembrane potential crosses the value $v_{\mathrm{m}}=v_{\text {gate }}$ with a negative time derivative. In figure $7-\mathrm{A}$, we plot the $l_{2, \mathrm{r}}$ norm of the error on the repolarisation time, for both solutions, while in figure 7 - B we plot the $A P D$ duration for the monodomain model (black line), for the standard eikonal model (red line) and for the eikonal model with $C V$ adaptation (blue line). The improved accuracy of the eikonal model with $C V$ adaptation depends on the improved accuracy in reproducing the $D I$ to a given 


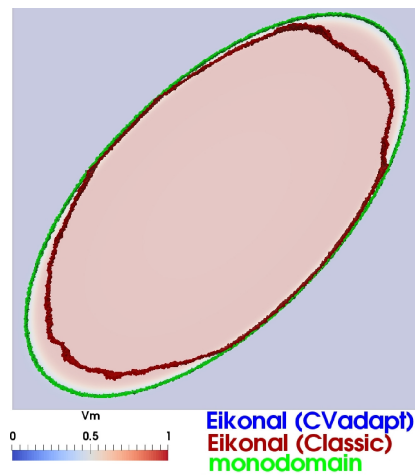

Time: $2050 \mathrm{~ms}$

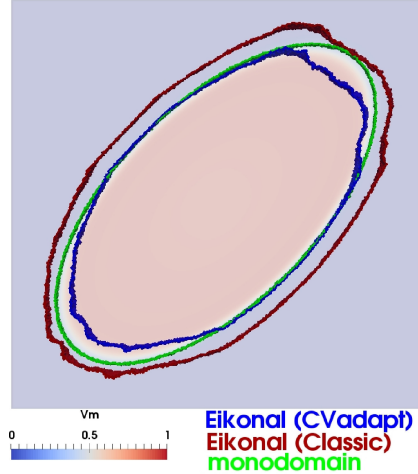

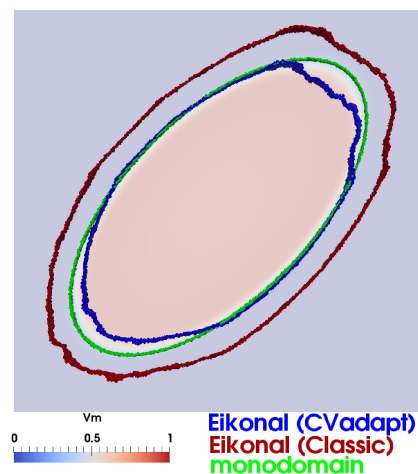

Time: $2450 \mathrm{~ms}$

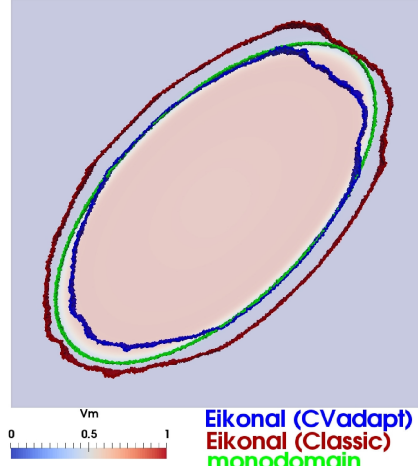

Figure 5: Oblique fibre direction Plot of the depolarization front of the monodomain solution (green dots), the standard eikonal solution (red dots) and the eikonal solution with CV adaptation (blue dots). The depolarization fronts are plotted at times $t=50,450,2050$ and 2450 ms. 


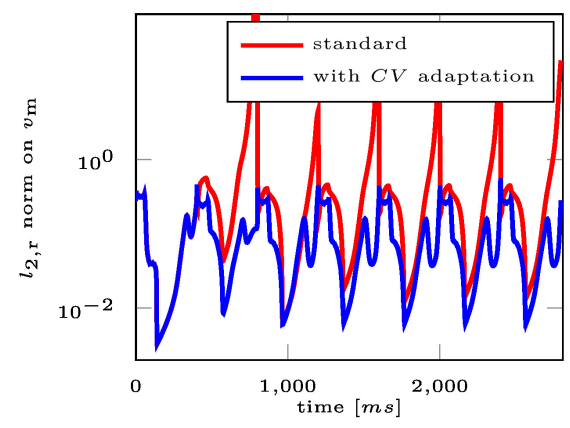

A

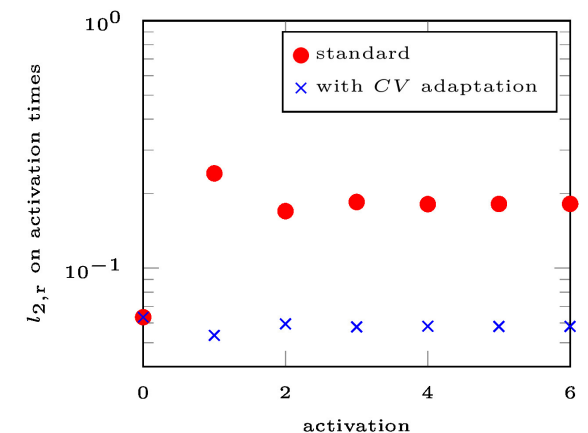

$\mathrm{B}$

Figure 6: A: Semi-logarithmic plot of the time course of the $l_{2, \mathrm{r}}$ error on the transmembrane potential for the standard eikonal model (red line) and for the eikonal model with $C V$ adaptation (blue line). B: Semi-logarithmic plot of the relative $l_{2, \mathrm{r}}$ error on the activation times at each stimulation for the standard eikonal model (red line) and for the eikonal model with $C V$ adaptation (blue line).

pacing rate.

\subsection{4. $C P U$ cost}

In this paragraph, we compare the CPU times required by algorithm 3 and algorithm 4 with respect to the CPU time required by the computation of the monodomain problem. The CPU time is evaluated on the example described in section 3.1 .1 for a single stimulus and until $T=400 \mathrm{~ms}$. We recall that the number of vertices in the mesh is 325,022 , the number of tetrahedra is $1,539,099$ and the used time step is $\mathrm{dt}=0.01 \mathrm{~ms}$. To better compare the computational performances, we do not consider the writing time. Simulations were conducted using an Intel Core i5 $2.9 \mathrm{GHz}$ processor. Monodomain simulations required $\approx 6$ hours (of which $\approx 5$ hours and 15 minutes for solving the linear system) to evaluate the the solution, while algorithm 3 required 7 minutes and 36 seconds (of which 7 minutes and 26 seconds required for the computing) and algorithm 4 spent 7 minutes (of which 6 minutes and 50 seconds required for the computing). Both eikonal algorithms revealed to be $\approx 50$ times faster compared to solving the monodomain problem. Algorithm 4 is $\approx 9 \%$ faster than algorithm 3 . The 


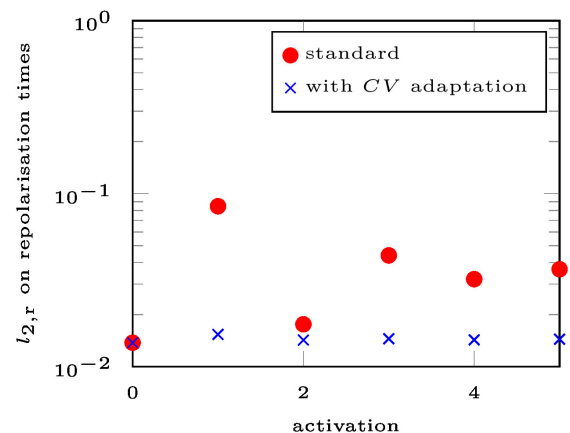

A

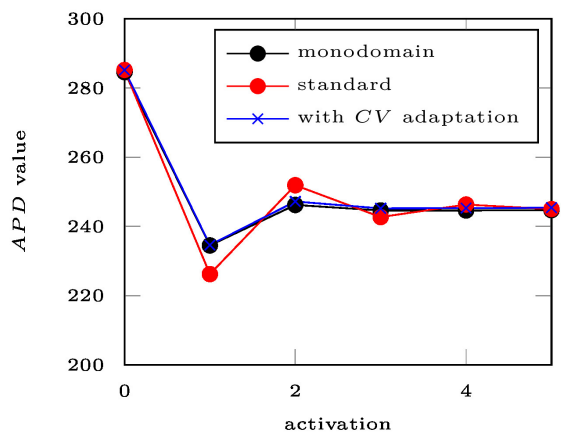

B

Figure 7: A: Semi-logarithmic plot of the relative $l_{2, \mathrm{r}}$ error on the repolarisation times at each stimulation for the standard eikonal model (red line) and for the eikonal model with $C V$ adaptation (blue line). B: Plot of the relative $A P D$ at each stimulation for the monodomain solution (black line), the standard eikonal model (red line) and for the eikonal model with $C V$ adaptation (blue line).

MS ionic model is described by a system of 2 differential equations; as far as the gate variable is concerned, we update its value at a new time step as follows:

$$
\mathrm{h}^{n+1}=\left\{\begin{array}{cc}
1+\left(\mathrm{h}^{n}-1\right) e^{-\frac{\mathrm{dt}}{\tau_{\mathrm{open}}}} & v_{\mathrm{m}} \leq v_{\text {gate }} \\
\mathrm{h}^{n} e^{-\frac{\mathrm{dt}}{\tau_{\text {close }}}} & v_{\mathrm{m}}>v_{\text {gate }}
\end{array}\right.
$$

where the exponentials can be evaluated once and offline. The update thus involves operations that are not expensive in terms of CPU time. The chosen time step allows updating the transmembrane potential with an explicit scheme that does not require sub-iterations. This factors yield a reduction of the computational overhead related to the solution of the ionic model in algorithm 3.

\subsubsection{A failure to propagate case}

The accuracy of the leading order approximation (2) for $A P D$ depends on the quantity $\frac{\tau_{\text {out }}}{\tau_{\text {close }}}$, as described in Mitchell \& Schaeffer (2003); when this quantity is not sufficiently small, the analytical expression underestimates the value of $A P D$. As depicted in Figure 5 in the original paper Mitchell \& Schaeffer (2003), when $\frac{\tau_{\text {out }}}{\tau_{\text {close }}} \geq 0.08$ the analytical expression evaluates an APD that is $20 \%$ 
shorter than its real value.

In this paragraph, we present an example obtained on the geometry presented in Figure 1, discretised with a regular mesh with size $\mathrm{h}=500 \mu \mathrm{m}$ and fibres $\vec{a}$ directed along the $x$ direction. The tissue is stimulated on the left edge of the slab, in the region $x \leq 0.25 \mathrm{~cm}$. We stimulate the tissue periodically with a basic cycle length of $200 \mathrm{~ms}$. The duration of the stimulus is $0.4 \mathrm{~ms}$ and its intensity is $I_{\mathrm{app}}=5 \mathrm{~ms}^{-1}$. In this simulation, we choose the following values for the ionic model parameters: $\tau_{\text {in }}=0.6 \mathrm{~ms}, \tau_{\text {out }}=12 \mathrm{~ms}, \tau_{\text {open }}=130 \mathrm{~ms}$, $\tau_{\text {close }}=80 \mathrm{~ms}$ and for the conductivities $\sigma_{\mathrm{m}}^{l}=2.0 \mathrm{~cm}^{2} / \mathrm{s}$ and $\sigma_{\mathrm{m}}^{t}=0.4 \mathrm{~cm}^{2} / \mathrm{s}$. Since $\frac{\tau_{\text {out }}}{\tau_{\text {close }}}=0.15$, this choice of parameters introduces an underestimation on $A P D$, when evaluated with the expression (2) and thus an overestimation of the $D I$, which affects the $C V$.

In the first row of Figure 8, we show snapshots of the numerical solution obtained by solving the monodomain problem at times 201, 240, 360, 401, $500 \mathrm{~ms}$. In the second row of the same figure, we show the numerical solution obtained with algorithm 3 for the same time values. Both solutions show a propagation failure when a stimulus is applied at $t=200 \mathrm{~ms}$. On the contrary, the stimulus applied at time $t=200 \mathrm{~ms}$ provides an electrical wave propagation if we use Algorithm 4 (Figure 8, third row). The reason is that the diastolic interval is overestimated using the analytical formula.

When a stimulus is applied at $t=400 \mathrm{~ms}$, all the three algorithms provide a new propagating electrical wave. However, the conduction velocity is underestimated using algorithm 4 compared to the monodomain solution and to algorithm 3 . The failure to propagate occurred at $t=200 \mathrm{~ms}$ produced a $D I$ that ranges on two basic cycle lengths for the monodomain and for algorithm 3 and thus a $C V$ faster than that evaluated by algorithm 4 .

\subsection{Heart geometry}

The geometrical model used in this work was derived from Computerized Tomography (CT) scan data Cardone-Noott et al. (2016) of the human heart. The initial ventricular mesh, generated with an edge length $\mathrm{h} \approx 400 \mu \mathrm{m}$ and contain- 

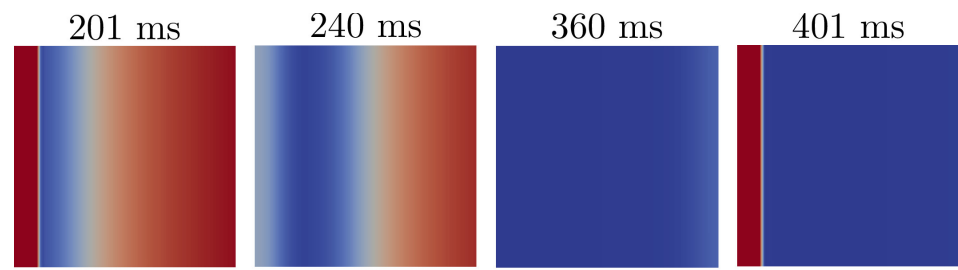

$500 \mathrm{~ms}$
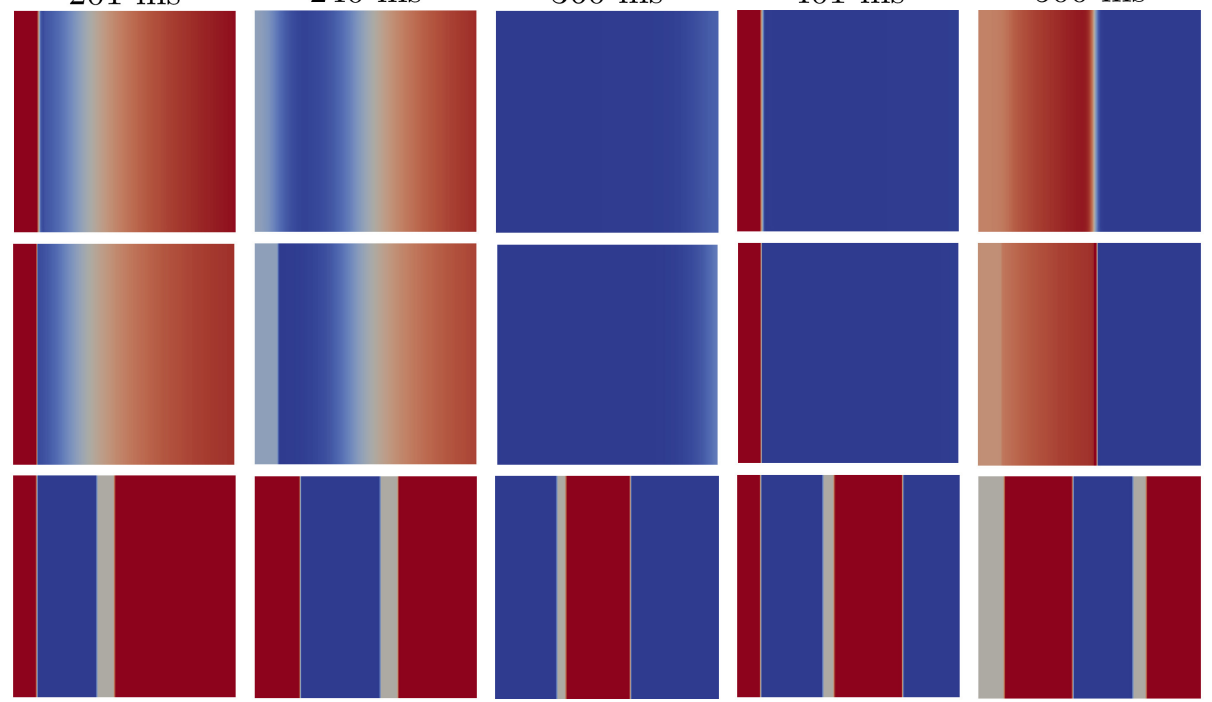

Figure 8: Action potential obtained solving the monodomain model (first row), Algorithm 3 (second row) and Algorithm 4 (third row). Snapshots are given at times $\mathrm{t}=201,240,360$, 401, $500 \mathrm{~ms}$.

ing 2.51 million nodes and 14.2 million tetrahedral elements, was re-sampled to $\approx 50,000$ nodes and $\approx 238,000$ tetrahedral elements. The computational mesh is depicted in Figure 9 - A. The fibres and the sheet orientations in the left and

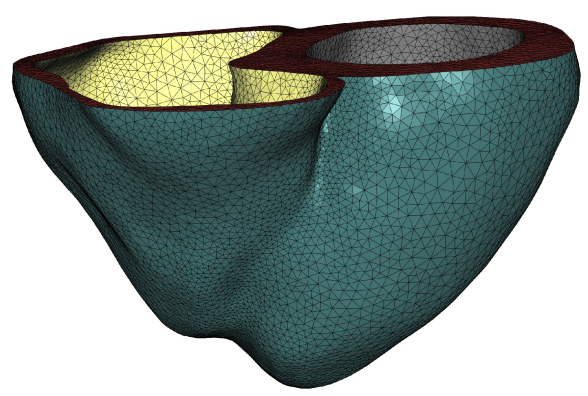

A

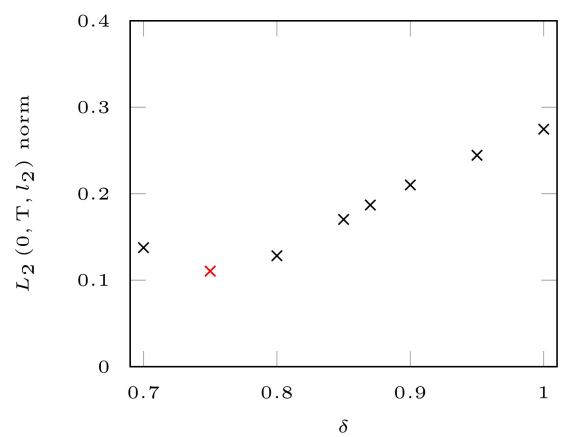

$\mathrm{B}$

Figure 9: A: Heart geometry discretized using a tetrahedral finite element mesh. B: Plot of the $L_{2}\left(0, \mathrm{~T}, l_{2}\right), T=400 \mathrm{~ms}$ norm of the error on the transmembrane potential with respect to the monodomain solution for several values of $\delta$. 
right ventricles are depicted in Figure 10 and were generated with the Streeter method Streeter et al. (1979); this method takes into account of the continuous change of the fibre angle across the ventricular wall. The myocardial tissue is characterised by a longitudinal conductivity $\sigma_{\mathrm{m}}^{l}=1.5 \mathrm{~cm}^{2} / \mathrm{s}$ and a transversal conductivity $\sigma_{\mathrm{m}}^{t}=0.15 \mathrm{~cm}^{2} / \mathrm{s}$.
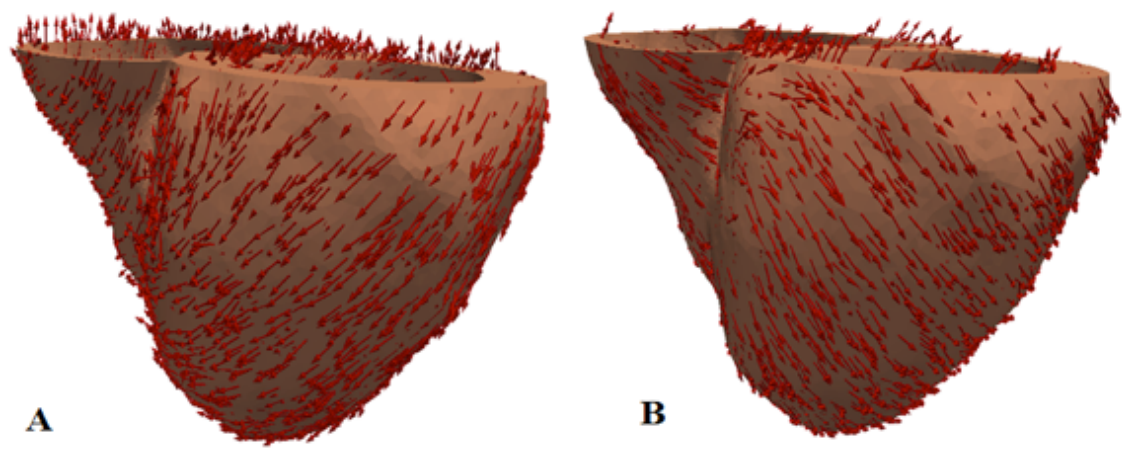

Figure 10: Streeter model generated (A) fibre and (B) sheet direction vectors, mapped on the ventricular geometry.

In this example, an external stimulus is applied at the apex of the left and the right ventricles and with an intensity $I_{\text {app }}=10 \mathrm{~ms}^{-1}$; the tissue is periodically stimulated by 5 stimuli at a basic cycle length of $400 \mathrm{~ms}$. The problem is discretized in time with a time step $\mathrm{dt}=0.01 \mathrm{~ms}$. The eikonal model is solved on the same mesh and with the same time step, and with a corrective coefficient $\delta=$ 0.75. This value was determined by first running the eikonal model within the time interval $[0,400] \mathrm{ms}$ for several values of $\delta$, then evaluating the $L_{2}\left(0, \mathrm{~T}, l_{2}\right)$ norm of the absolute error on the transmembrane potential for each value of $\delta$ and finally choosing the value yielding the minimum $L_{2}\left(0, \mathrm{~T}, l_{2}\right)$ norm of the error. In Figure 9 - B, we plot the $L_{2}\left(0, \mathrm{~T}, l_{2}\right)$ norm for several values of $\delta$; in the same figure, the optimal value $\delta=0.75$ is marked in red. In Figure 11 - A, we plot the time course of the $l_{2, \mathrm{r}}$ error on the transmembrane potential for the standard eikonal model (red line) and the eikonal model with $C V$ adaptation (blue line). When the first stimulus is applied, the tissue is initially at rest, thus 


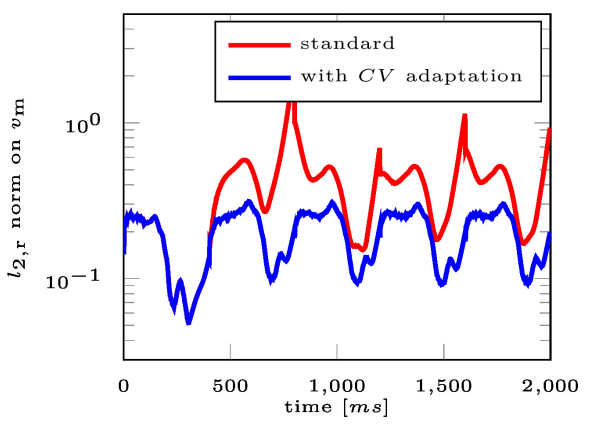

A

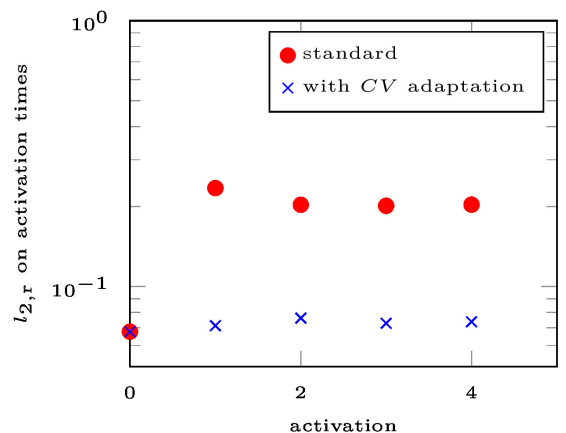

B

Figure 11: A: Semi-logarithmic plot of the time course of the $l_{2, r}$ error on the transmembrane potential for the standard eikonal model (red line) and for the eikonal model with $C V$ adaptation (blue line). B: Semi-logarithmic plot of the relative $l_{2, \mathrm{r}}$ error on the activation times at each stimulation for the standard eikonal model (red line) and for the eikonal model with $C V$ adaptation (blue line).

$D I \rightarrow \infty$, the value of $C V$ is equal to its maximum and both the models produce the same error. When the subsequent stimuli are applied, $\mathrm{h}<1$, the $D I$ has a finite value and the $C V$ adapts according to (6); thus, the eikonal model with the $C V$ adaptation produces a significantly smaller error compared to the error obtained by solving the standard eikonal model. Figure 11 -B shows the $l_{2, \mathrm{r}}$ error on the activation times for each of the 5 cycles. Along the 5 cycles, the mean of the $l_{2, \mathrm{r}}$ error on the activation times is $7 \%$ when using the eikonal model with $C V$ adaptation, while it is $18 \%$ when using the standard eikonal model. In Figure 12, the depolarization fronts are plotted for the monodomain model (green dots), the standard eikonal model (red dots) and the eikonal model with $C V$ adaptation (blue dots). The depolarization front is tracked by extracting the surfaces where $v_{\mathrm{m}}=v_{\text {gate }}$. The figure highlights the improvements on the accuracy of the eikonal model when the $C V$ is adapted to the heart rate. A video showing the comparison of the depolarization fronts is available in the online supplement. 

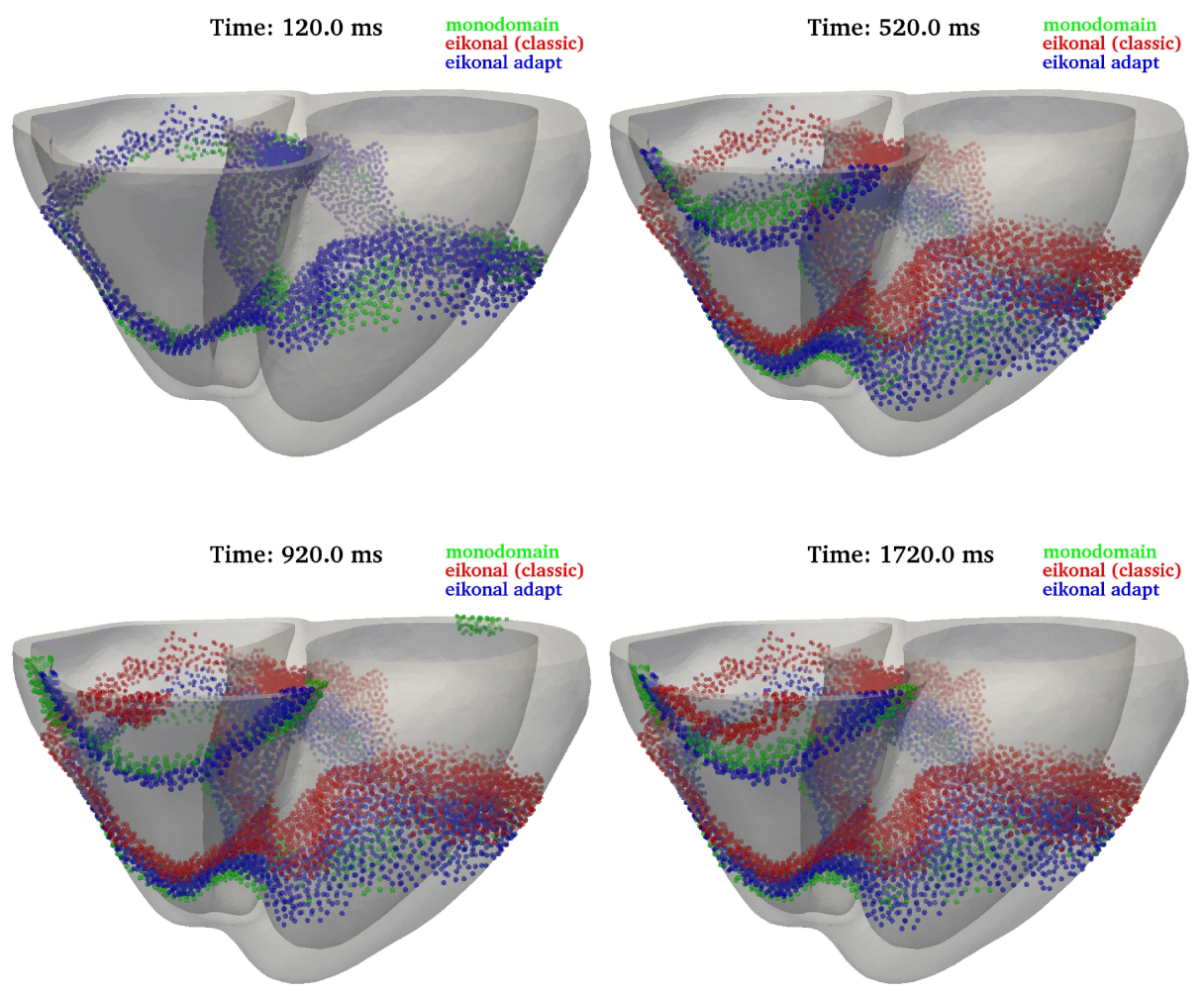

Figure 12: Depolarization fronts at $\mathrm{t}=120,520,920,1720 \mathrm{~ms}$ for the monodomain model (green dots), the standard eikonal model (red dots) and the eikonal model with $C V$ adaptation (blue dots). The depolarization fronts are tracked as the surfaces where $v_{\mathrm{m}}=v_{\text {gate }}$.

\section{Discussion}

The algorithm proposed in this paper allows generating a locally personalised computational model during the clinical procedure and thus guiding the radiofrequency ablation, when coupled with an efficient algorithm for the local characterisation of tissue properties, like the one described in Corrado et al. (2016).

Unlike the algorithm introduced in Pernod et al. (2011); Wallman et al. (2013), the eikonal model presented in this paper also takes into account of the local changes in the characteristic propagation velocity and thus is suitable for 
the simulations of the multi-front propagation and multi-stimuli applications, in particular for the description of the activation pattern characteristic of heart tachyarrhythmias. In this paper, we adopted the leading order approximations presented in Mitchell \& Schaeffer (2003); Cain et al. (2004) for the APD and the CV; notwithstanding that, the approach here presented can be extended to any characterisation of the $C V$ as a function of the history of the $D I$, the $A P D$, or any other quantity related to the computed solution. For instance, the current model can be adopted in the applications presented in Pernod et al. (2011); Wallman et al. (2013), producing a solution significantly closer to the monodomain model. The effects of the $C V$ adaptation become significant when the $D I$ decreases. Thus, the improvement is significant especially at high heart rate conditions or in presence of frequently activated regions in the heart tissue.

The solution of the ionic model is used to determine if a node in the graph is re-excitable and thus allows to reproduce the absolute refractory period (ARP). The solution of the eikonal model provides only the time the activation front reaches a node in the graph, while the intensity of the activation current is kept constant and homogeneous within the domain. Since the intensity of the depolarization front affects the propagation of the electrical stimulus during the effective and the relative refractory periods (ERP, RRP), this approximation may impact the ability and the accuracy of the model to capture propagation failures during refractoriness. A possible solution to overcome this limitation is the matter of a future work. The leading order estimate of the $C V$ restitution, obtained in Cain et al. (2004) and here used to characterise the front propagation velocity, refers to a steady state equilibrium reached when the tissue is stimulated at a constant rate and for a sufficiently long time. Since the MS model does not take into account any memory effect, the $C V$ adapts instantaneously to the new pacing condition. However, since we lose the diffusion term in the eikonal model with or without $C V$ adaptation, it is more difficult to accurately simulate re-entry waves. The main issue is that the curvature of the wavefront is not well estimated with this model. Hence, we don't recommend 
using this model to study re-entry and in particular spiral waves. Due to the absence of a diffusive term, the eikonal model may compromise the accuracy of the repolarisation wave when a high APD gradient is present in the tissue. A possible solution that may overcome this issue is recently proposed in Neic et al. (2017) where the authors adopt an eikonal solver to compensate the error on the conduction velocity when a coarse mesh is used to solve the monodomain equation. This approach, however, does not take into account of the dependency of $\mathrm{CV}$ on the pacing rate and its variations. The Dijkstra algorithm is lighter in terms of computational cost and less complex in terms of implementation compared to a fast-marching scheme, Pernod et al. (2011). However, the Dijkstra algorithm computes the activation times following the shortest pathway in the graph built using the edges of the mesh; this leads to an accuracy loss when following the graph edges. This source of inaccuracy in the Dijkstra solution produces an irregular propagation front, delayed in the regions where the curvature is highest as shown in Figures 2, 4 and 5. This is not the case of some fast marching schemes, that allow taking into account of anisotropy, Pernod et al. (2011); Sermesant et al. (2007).

\section{Conclusions}

We presented a computational model combining the eikonal equation and the Mitchell and Schaeffer ionic model for solving the propagation of the electrical wave in the heart. This algorithm adapts the conduction velocity of the eikonal model to the electrophysiological state and the heart rate through the analytical $C V$ restitution expression and a locally computed $D I$ allowing an accurate simulation of multi-front activations. This algorithm provides a reasonable trade-off between computational time and accuracy. Like the monodomain equations, the model here developed allows computing the transmembrane potential and the state variables of the ionic model. Using 3D numerical simulations, we have shown that this model improves the accuracy of tracking the position of the wavefront compared to the standard eikonal method. 
We think that this new formulation of the eikonal model is suitable for clinical applications since it requires fewer computational time and resources than the monodomain and the bidomain models and shows a better accuracy than the standard eikonal model.

\section{References}

Aliev, R., \& Panfilov, A. (1996). A simple two-variable model of cardiac excitation. Chaos, Solitons and fractals, 7, 293-301.

Cain, J. W., Tolkacheva, E. G., Schaeffer, D. G., \& Gauthier, D. J. (2004). Ratedependent propagation of cardiac action potentials in a one-dimensional fiber. Physical review E, 70, 061906.

Cardone-Noott, L., Bueno-Orovio, A., Mincholé, A., Zemzemi, N., \& Rodriguez, B. (2016). Human ventricular activation sequence and the simulation of the electrocardiographic qrs complex and its variability in healthy and intraventricular block conditions. Europace, 18, iv4-iv15.

Clements, J., Nenonen, J., Li, P., \& Horacek, B. (2004). Acivation dynamics in anisotropic cardiac tissue via decoupling. Annals of Biomedical Engineering, 2, 984-990.

Corrado, C., Gerbeau, J.-F., \& Moireau, P. (2015). Identification of weakly coupled multiphysics problems. application to the inverse problem of electrocardiography. Journal of Computational Physics, 283, 271-298.

Corrado, C., Lassoued, J., Mahjoub, M., \& Zemzemi, N. (2016a). Stability analysis of the $\{P O D\}$ reduced order method for solving the bidomain model in cardiac electrophysiology. Mathematical Biosciences, 272, 81 - 91. doi:http://dx.doi.org/10.1016/j.mbs.2015.12.005.

Corrado, C., Whitaker, J., Chubb, H., William, S., Wright, M., Gill, J., O’Neill, M., \& Niederer, S. (2016b). Personalized models of human atrial electro- 
physiology derived from endocardial electrograms. IEEE Transactions on Biomedical Engineering, PP, 1-1. doi:10.1109/TBME.2016.2574619.

Ethier, M., \& Bourgault, Y. (2008). Semi-implicit time-discretization schemes for the bidomain model. SIAM J. Numer. Anal., 46, 2443-2468.

Kim, J., \& Hespanha, J. P. (2003). Discrete approximations to continuous shortest-path: Application to minimum-risk path planning for groups of uavs. In Decision and Control, 2003. Proceedings. 42nd IEEE Conference on (pp. 1734-1740). IEEE volume 2.

Luo, C.-h., \& Rudy, Y. (1991). A model of the ventricular cardiac action potential. depolarization, repolarization, and their interaction. Circulation research, 68, 1501-1526.

Luo, C.-h., \& Rudy, Y. (1994). A dynamic model of the cardiac ventricular action potential. i. simulations of ionic currents and concentration changes. Circulation research, 74, 1071-1096.

Mitchell, C., \& Schaeffer, D. (2003). A two-current model for the dynamics of cardiac membrane. Bulletin Math. Bio., 65, 767-793.

Neic, A., Campos, F. O., Prassl, A. J., Niederer, S. A., Bishop, M. J., Vigmond, E. J., \& Plank, G. (2017). Efficient computation of electrograms and ecgs in human whole heart simulations using a reactioneikonal model. Journal of Computational Physics, 346, 191 - 211. doi:http://dx.doi.org/10.1016/j.jcp.2017.06.020.

Pathmanathan, P. et al. (2012). Computational modelling of cardiac electrophysiology: explanation of the variability of results from different numerical solvers. International journal for numerical methods in biomedical engineering, 28, 890-903.

Pernod, E., Sermesant, M., Konukoglu, E., Relan, J., Delingette, H., \& Ayache, N. (2011). A multi-front eikonal model of cardiac electrophysiology for in- 
teractive simulation of radio-frequency ablation. Computers 83 Graphics, 35, 431-440.

Roberts, D. E., Hersh, L. T., \& Scher, A. M. (1979). Influence of cardiac fiber orientation on wavefront voltage, conduction velocity, and tissue resistivity in the dog. Circulation Research, 44, 701-712.

RUSH, S., ABILDSKOV, J. A., \& MCFEE, R. (1963). Resistivity of body tissues at low frequencies. Circulation Research, 12, 40-50. doi:10.1161/01.RES.12.1.40.

Sermesant, M., Konukoglu, E., Delingette, H., Coudière, Y., Chinchapatnam, P., Rhode, K. S., Razavi, R., \& Ayache, N. (2007). An anisotropic multi-front fast marching method for real-time simulation of cardiac electrophysiology. In F. B. Sachse, \& G. Seemann (Eds.), Functional Imaging and Modeling of the Heart: 4th International Conference, FIHM 2007, Salt Lake City, UT, USA, June 7-9, 2007. Proceedings (pp. 160-169). Berlin, Heidelberg: Springer Berlin Heidelberg.

Sethian, J. A. (1996). A fast marching level set method for monotonically advancing fronts. Proceedings of the National Academy of Sciences, 93, 15911595.

Streeter, D., Berne, R., Sperelakis, N., \& Geiger, S. (1979). Gross morphology and fiber geometry of the heart. Handbook of Physiology, Section 2: The Cardiovascular System, 1, 61-112.

Ten Tusscher, K., Noble, D., Noble, P., \& Panfilov, A. (2004). A model for human ventricular tissue. American Journal of Physiology-Heart and Circulatory Physiology, 286, H1573-H1589.

Tung, L. (1978). A bi-domain model for describing ischemic myocardial $D-C$ potentials. Ph.D. thesis MIT.

Wallman, M., Bueno-Orovio, A., \& Rodriguez, B. (2013). Computational probabilistic quantification of pro-arrhythmic risk from scar and left-to-right het- 
erogeneity in the human ventricles. In Computing in Cardiology Conference (CinC), 2013 (pp. 711-714). IEEE.

Wallman, M., Smith, N. P., \& Rodriguez, B. (2012). A comparative study of graph-based, eikonal, and monodomain simulations for the estimation of cardiac activation times. Biomedical Engineering, IEEE Transactions on, 59, $1739-1748$. 